\title{
eCushion: An eTextile Device for Sitting Posture Monitoring
}

\author{
Wenyao $\mathrm{Xu}^{13}$, Zhinan $\mathrm{Li}^{2}$, Ming-Chun Huang ${ }^{1}$, Navid Amini ${ }^{13}$ and Majid Sarrafzadeh ${ }^{13}$ \\ 1. Electrical Engineering Department, University of California, Los Angeles \\ 2. Electrical Engineering Department, Peking University, China \\ 3. Wireless Health Institute, University of California, Los Angeles
}

\begin{abstract}
Sitting posture analysis is critical for daily applications in biomedical, education and healthcare fields. However, it remains unclear how to monitor sitting posture economically and comfortably. To this end, we presented an eTextile device, called eCushion, in this paper, which can analyze the sitting posture of human being accurately and non-invasively. First, we discussed the implementation of eCushion and design challenges of sensing data, such as scale, offset, rotation and crosstalk. Then, several effective techniques have been proposed to improve the recognition rate of sitting posture. Our experimental results show that the recognition rate of our eCushion system could achieve $92 \%$ for object-oriented cases and $79 \%$ for general cases.
\end{abstract}

\section{INTRODUCTION}

Sitting is one of the nature postures of human beings. Reported by CNN, most of people sit six hours per day on average in daily lives. Nowadays, sitting posture analysis becomes an important medical research topic due to its extensive applications with significant impact in many domains, such as biomedical (rehabilitation evaluation in chronic diseases) [1], health education (correct sitting posture for back ache prevention) [2], human computer interface (real-time sitting posture analysis for gaming) [3] and facilities design (furniture fitness evaluation) [4].

In order to monitor the sitting posture accurately and reliably, sensors are needed around the users to get the sitting features. Intuitively, camera is the most straightforward and comprehensive method. [5] deployed several cameras in a work station to monitor the sitting postures of workers. However, it always makes the users uncomfortable because it records other unnecessary information related to personal privacy at the same while. [6] uses pressure sensors [7] to equip chairs for sitting posture analysis. Compared to camera, pressure sensor is more economical and easier to be utilized. However, this kind of sensors is commonly manufactured out of piezoelectric materials such as quartz, which is invasive or uncomfortable for daily use.

In the past few years, the researchers had designed kinds of textile-based sensors, called eTextile, to measure pressure [8], stretch [9], temperature [10] and humanity [11]. Contrary to silicon or piezoelectric based sensors, eTextile is close to normal wearable fabric in terms of price and feeling. Therefore, it can be unobtrusively integrated into different apparels for daily applications. [12] presented a garment prototype using textile strain sensors to recognize upper body postures. [13] introduced an interactive system using a wearable textile sensor to monitor breathing patterns of human beings. [14] built textile pressure sensor arrays in a shoe for gait analysis.

However, the characteristics of eTextile are not ideal for sensing. Usually, the sensing values suffer from several instable factors such as noise, scaling, offset and crosstalk. Especially when it has been integrated into clothes or facilities, other more uncertainties will appear to make the signal more fuzzy. For instance, in our application, pressure distribution is not only related to sitting posture, but also depends on other factors such as the subject's size, weight and sitting orientation.

In this paper, we present an eTextile device, named eCushion, for sitting posture monitoring. First, we analyze the modeling of sensors and propose effective techniques to compensate the crosstalk in sensor arrays. Furthermore, we characterize sitting posture feature with pressure profile sequence to get rid of other variations such as noise, scaling and offset effects. Additionally, dynamic time warping based algorithm is proposed for phase-insensitive time series signal indexing. Our experimental results show that the recognition rate of eCushion for sitting posture could achieve up to $92 \%$.

The remainder of this paper is organized as follows. Section II presents eCushion system. Section III introduces sensor array design and challenging issues in sensing data. Section IV describes the algorithm for uncertainty reduction and pattern matching of signals. Experimental results are shown in Section V, and the paper concludes in Section VI.

\section{SySTEM DESCRIPTION}

eCushion is designed to use for both clinical trial in the hospital and daily use at home where the user can be remotely monitored. Therefore, the essential problem is how to make the system convenient to access, straightforward to operate and unobtrusive to deploy. Also, the system should be low-cost and compatible with existing computer systems found in hospitals or homes. Fig. 1 shows the implementation of eCushion. It comprises of a textile sensor array, signal sensing \& transferring unit and a computation \& display terminal . A 16 by 16 textile sensor array, shown in Fig. 2, is used to monitor the pressure distribution on cushion when users are sitting on it. The footprint of the sensor is $5 / 8$ inch by $5 / 8$ inch, and adjacent space is $1 / 8$ inch. An Arduinobased unit is in Fig. 1(b) to sample and transfer the sensing data via bluetooth protocol. For sitting posture monitoring application, the sample rate is set as $10 \mathrm{~Hz}$. Considering the accessibility for mobile users, as shown in Fig. 1(c), a 
user-friendly Graphical User Interface (GUI) is developed on smart phone which gives real-time feedback of sitting pressure distribution and facilitates continuous monitoring of the user sitting posture.

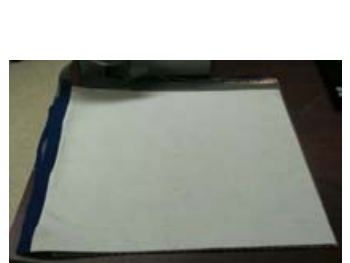

(a) Textile Sensor Array

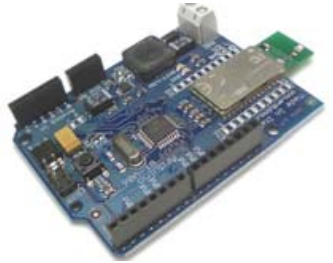

(b) Arduino + Bluetooth

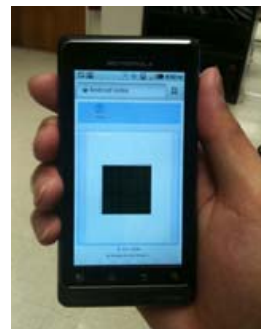

(c) Smart Phone

Fig. 1. eCushion System

\section{Sensor ARray Design}

eTextile [15] is a fiber-based yarn which is coated with piezoelectric polymer. And the initial resistor between the top-bottom surfaces is high. When extra force is applied on surface of the eTextile, the intra fibers will be squeezed together and the throughout resistor becomes smaller. Note that the resistor between any two points on the same side can be treated as constantly infinite. Therefore, we can take advantage of this characteristics and design a high-density and low-cost pressure sensor array.

\section{A. Sensor Array Architecture}

One type of textile sensor sheet has been implemented in [16]. In that structure, each sensor needs an independent ADC channel to sample the data. For example, to build a $N$ by $N$ sensor array, $N^{2}$ I/O pins are required, which is prohibitive for large-scale sensing problem. In eCushion, we still use three stacked-layer structure to build the sensor, where the middle layer is eTextile. Difference is that the top layer and the bottom layer are normal fabric uniformly coated with parallel conductive buses. As the structure shown Fig. 2(a), the conductive buses on the top layer are orthogonal to those on the bottom. The intersection part is the sensing unit. Fig. 2(b) shows the sensor implementation. The top layer and bottom layer are coated with 16 buses respectively. Therefore, the number of sensors located on this sheet is 256. Moreover, with the comparison in Fig. 2(c) the total thickness is $1.5 \mathrm{~mm}$ only, which enable eCushion flexible and thin enough for noninvasive use. As shown in Fig. 2(a), this $N$ by $N$ sensor structure only has $2 N$ I/O pins. To explain the operation of this sensor array, we will discuss the corresponding sensor scanning method in the next part.

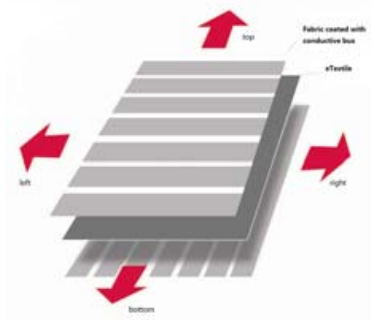

(a) Sensor Structure

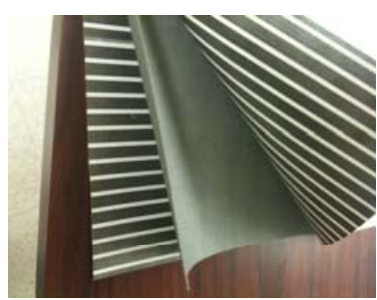

(b) Sensor Implementation

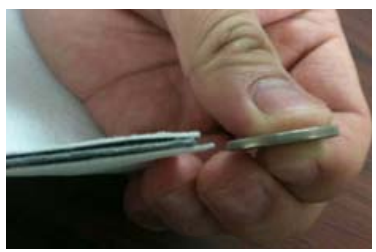

(c) Sensor Thickness

Fig. 2. Sensor Sheet

\section{B. Scanning Method}

The peripheral circuit shown in Fig. 3 is used to scan the zebra pattern sensor array. Each conductive bus on the bottom is connected to ADC via analog switch model $S_{1}$ and to ground via an offset resistor $R_{0}$; each conductive bus on the top is connected to voltage supply via analog switch model $S_{2}$. Both $S_{1}$ and $S_{2}$ are controlled by microcontroller. When one bus $i$ on the top is selected by $S_{2}$ to connect to voltage supply, the reading of channel $j$ in $S_{1}$ is the value of Sensor $V_{i j}$. With peripheral circuit, the microcontroller can access arbitrary sensor value in this array.

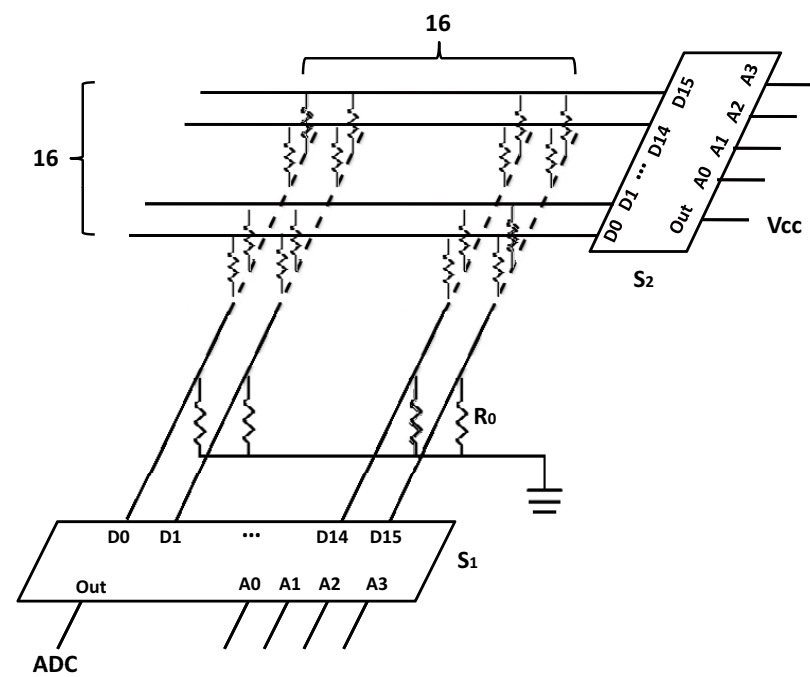

Fig. 3. Peripheral Circuit for Sensor Scanning

\section{Challenging of eTextile Sensing}

When the users sitting and applying forces on the sensor sheet, the eTextile output is not only depending on the sitting 
posture. In fact, the sensor value can be interfered by other uncertain issues. Here, we list and study all the dominant factors for signal distortion. Basically, there are four kinds of uncertain issues, where offset and scale are called sheet to sheet uncertainty, and crosstalk and rotation belong to within sheet uncertainty.

1) Offset

In ideal case, the initial pressure on each sensor is zero. However, due to the sandwiched structure, initial noise pressure or offset value is unavoidable and its value highly depends on the assemble method of the sensor. Assemble variation will apply different initial pressures from sheet to sheet. The tighter three layers are sandwiched, the larger the offset is applied.

2) Scale

The characteristics of eTextile have large process variation. When the same forces are applied to two eTextile sheets respectively, the sensor outputs are not necessarily the same. Therefore, it is impossible to find one Look-up table to generally describe the relationship between applied force and sensor output. However, eTextile sensor is still promising because the sensing repeatability and relativity are very stable.

3) Crosstalk

The crosstalk effect is one of the most tough issues to deal with. Due to the limited space, the adjacent sensors are mechanically coupled together. As shown in Fig. 4, the thickness of the sensor sheet is uniform, valued $H_{1}$, when the pressure is free. Once some force is applied on Sensor $S_{3}$, its thickness will be reduced to $\mathrm{H}_{2}$. In the mean while, the adjacent sensors also get sequeezed: the sensor values of $S_{2}$ and $S_{4}$ get changed along with $S_{3}$. However, it is still promising to compensate this effect since crosstalk effect is localized among neighboring units based on the experiments.
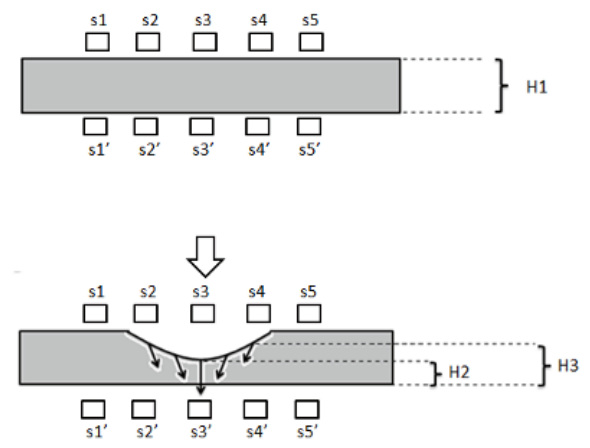

Fig. 4. Crosstalk effect in sensor array

4) Rotation

Even if the user sits on eCushion with the same gesture, the pressure map could still be largely different because of the sitting orientation. Fig. 5 shows two pressure distributions with the same gesture but different orientations. The pressure map in Fig. 5(a) is similar to that in Fig. 5(b). However, it is highly difficult to match them with each other using pattern recognition techniques because "rotation is always something hard to handle compared to translation and scaling" [17].

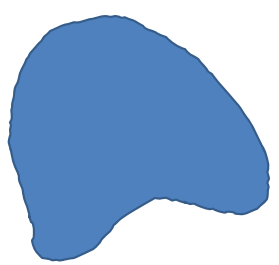

(a) Map One

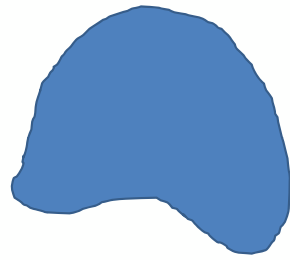

(b) Map Two
Fig. 5. Pressure map rotation

In this section, we presented the sensor design and imperfection of eTextile sensing. To achieve high recognition rate, the above listed issues should be addressed effectively before performing pattern recognition. In the next section, we will introduce the proposed techniques to improve the sensor data for accurate sitting posture recognition.

\section{Sitting Posture Recognition}

In this section, we will discuss sitting posture recognition algorithm based on pressure distribution. [18] proposed a template-based algorithm to distinguish postures, but it cannot handle the scenario where the sensing data is imperfect. [11] used Naive Bayes Network [19] to train the data and selected significantly featured sensors for classification. However, this method highly depends on the training data and cannot deal with the variational factors such as different weights, sizes and sitting orientation. To address the challenging issues of textile, we proposed an optimization algorithm to compensate the signal for accurate signal classification.

\section{A. Data Preprocessing for Crosstalk}

Crosstalk is a cluster-based effect and related to the whole data, which should be first dealt with. Assuming that there are $n$ pressure sensors in eCushion, the crosstalk effect among $n$ sensors can be expressed as:

$$
F_{1 \times n} C_{n \times n} \doteq D_{1 \times n}
$$

where $D_{1 \times n}$ denotes $n$ sensing values, $F_{1 \times n}$ denotes the force applied on $n$ sensors, and $C_{n \times n}$ denotes $\mathrm{F}$ to $\mathrm{D}$ transformation matrix including the crosstalk effect. It means whenever $f_{i}$ applies on sensor $i$, the impact on sensor $j$, denoted as $A_{i j}$ can be calculated by:

$$
f_{i} c_{i j}=A_{i j}
$$

Therefore, the value of sensor $j$, denoted as $d_{j}$, can be calculated as:

$$
d_{j}=\sum A_{i j}
$$

To investigate the crosstalk, we did preliminary experiments. We applied unit force on single sensor $i$, denoted as 
$F_{e i}$, and measured the output value from each senor $D_{e i}$ which is $1 \times n$ vector. Based on Equ. 1, we get as follows:

$$
C_{n \times n}=\left[\begin{array}{c}
D_{e 1} \\
D_{e 2} \\
\cdot \\
\cdot \\
\cdot \\
D_{e n}
\end{array}\right]
$$

Based on experiments, we found transformation matrix $C_{n \times n}$ is sparse, and all elements on the diagonal are nonzero. Therefore, the inverse matric of $C_{n \times n}$ can be easily calculated, called decoupling matrix $C^{-1}$. We can obtain pressure map without crosstalk by the following formula:

$$
F_{1 \times n} \doteq D_{1 \times n} C_{n \times n}^{-1}
$$

\section{B. Data Representation}

To facilitate the pattern recognition, it is significant to choose an appropriate way for data representation. Rather than dealing with pressure map (2D image) directly, we convert it into pressure profile sequence (1D time series). The procedure is as follows:

1) With pressure map canny edge detector is applied to the pressure map to obtain a binary image.

2) Extracting the outline curve of the binary image.

3) Measuring the distance between every point and image center which can be treated as the Y-axis of a sequential signal.

Fig. 6 shows the procedure of this conversion. With sequential signal, some distortions, including offset and scaling, could be easily eliminated through z-normalization:

$$
x_{n}^{\prime}=\frac{x_{n}-\mu}{\sigma}
$$

where $\mu$ is the signal expectation value, and $\sigma$ is the signal variance.

Moreover, sequential signal could leakage some existing methods to deal with rotation problem in pressure map, which will be detailed in the next subsection.

\section{Signal Matching using Dynamic Time Warping}

We use dynamic time warping as pattern recognition methods to classify different sitting postures. The targeted sequential signals are extracted from the corresponding pressure map while user is sitting on eCushion. Dynamic time warping $(D T W)$ is a similarity evaluation for two time series signals. Compared to Euclidean distance, $D T W$ is more robust, allowing similar shapes to be matched even if they are out of phase [20].

Assuming there are two pressure profile sequences:

$$
\begin{aligned}
& S=\left[s_{1}, s_{2}, s_{3}, \cdot \cdot, s_{i}, \cdot \cdot s_{n}\right] \\
& T=\left[t_{1}, t_{2}, t_{3}, \cdot \cdot t_{j}, \cdot \cdot t_{m}\right]
\end{aligned}
$$

To evaluate the similarity of these two sequences, $D T W$ constructs a $n$ by $m$ matric $D$, where $d_{i j}=\left(s_{i}-t_{j}\right)^{2}$.

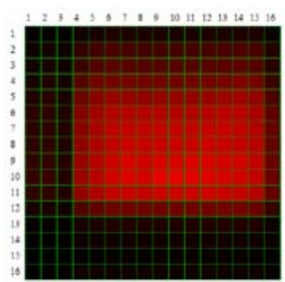

(a) Map

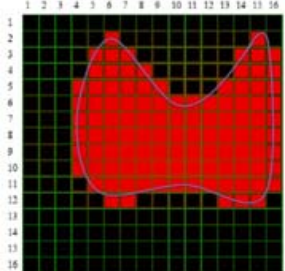

(b) Boundary

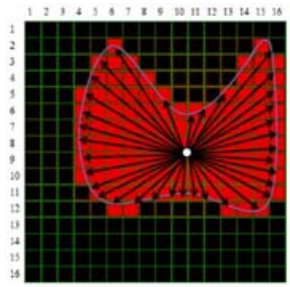

(c) Distance

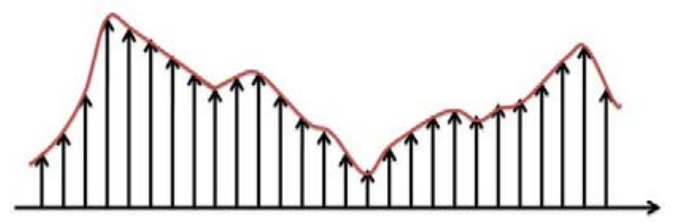

(d) Pressure Profile Sequence

Fig. 6. Data Representation Procedure

Each element $d_{i j}$ denotes the similarity between $s_{i}$ and $t_{j} . D T W$ is to find a continuous and monotonic path $W$ from $d_{11}$ to $d_{m n}$ with minimal cost. The time and space complexity of $\operatorname{DTW}(S, T)$ is $\Theta(m n)$. Fig. 7 shows an example of $D T W$-based similarity evaluation between two pressure profile sequences.

We use $L B_{-} K \operatorname{eogh}[21]$ to speed up the algorithm. Given any $2 r$-length subsequence $S \prime=\left[s_{i-r}, s_{i+r}\right]$ in $\mathrm{S}$, we can find the upper bound of $S \prime$ is $U_{i}$, and the lower bound of $S \prime$ is $L_{i}$. Therefore, $\operatorname{DTW}(S, T)$ with $L B \_K \log h$ is:

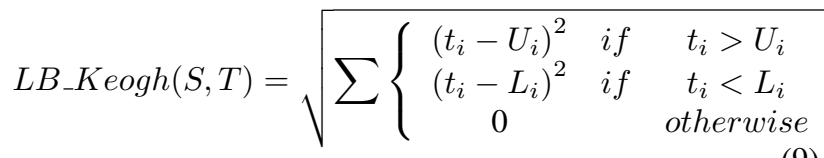

Notice that $L B \_K e o u g h$ will regress to Euclidean distance when $r$ is 1 . This strategy dramatically speed up DTW-based indexing [21]. 


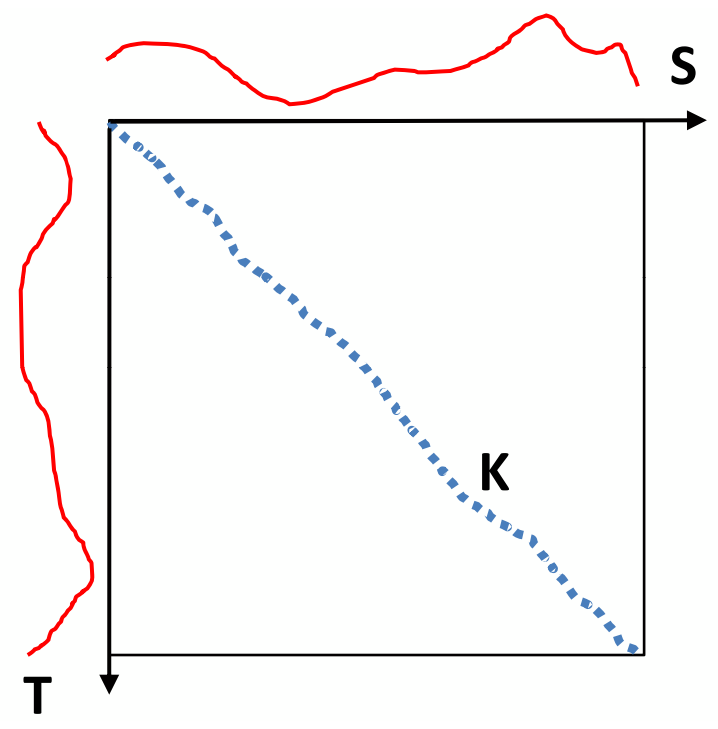

Fig. 7. A warping example of two pressure profile sequences

TABLE I

EXPERIMENTAL RESULTS

\begin{tabular}{|c|c|c|}
\hline & self training & general training \\
\hline accurate rate & $92 \%$ & $79 \%$ \\
\hline
\end{tabular}

\section{EXPERIMENTS}

\section{A. Experimental Setup}

To evaluate the accuracy of our eCushion system, we performed a pilot on campus. There are ten objects, including six male and four female.

Each participant sits on eCushion with seven predesigned sitting postures for five rounds. These data are used as training data. The seven postures are considered to classify, including 1) situp, 2) forward, 3) backward, 4) left lean, 5) right lean, 6) right foot over left and 7) left foot over right.

Fig. 8 shows an example to explain the procedure of data acquisition and preprocessing. The figures on the top row illustrates seven sitting postures. The middle row pictures show the corresponding pressure map of each posture. Each pressure map has total 256 pixels. The bottom sequences are the extracted pressure profile sequences from pressure maps. Each sequence is evaluated and classified with DTW.

\section{B. Recognition Results}

Two evaluation methods are performed in sitting posture analysis. The first is the pattern recognition based on self training data. As shown in Table $\mathrm{I}$, the recognition rate on self-training could achieve $92 \%$. The second method is the evaluation with general training data. This evaluation method is more fair and objective. In Table I, it shows that the correction rate for posture classification is $79 \%$ by average. Our experimental results illustrates that our designed eCushion system could safely achieve a high classification rate.

\section{CONCLUSION AND FUture WORK}

In this paper, we designed and implemented eCushion for sitting posture monitoring. Towards several challenging issues in eTextile sensor, including scaling, offset, crosstalk, and rotation effects, we proposed an optimized strategy to compensate the signal for accurate signal analysis. Experiments show that eCushion could achieve a high sitting posture recognition rate, $79 \%$ for general cases and $92 \%$ for object-oriented cases. In the future, we will try to improve the recognition rate for general case. Meanwhile, due to the scalability of the eTextile device, we consider to further scale up our system for other applications such as bedsheet for sleeping monitoring and carpet for gait analysis.

\section{ACKNOWLEDGMENT}

The authors would like to thank Dr. Anpeng Huang in Peking University for his constructive suggestions.

\section{REFERENCES}

[1] S. Perlmutter, F. Lin, and M. Makhsous, "Quantitative analysis of static sitting posture in chronic stroke," Gait Modeling, vol. 10, pp. 13 - 17, Jun. 2010.

[2] S. Haynes and K. Williams, "Impact of seating posture on user comfort and typing performance for people with chronic low back pain," International Journal of Industrial Ergonomics, vol. 4, pp. 186 - 194, Jan. 2008.

[3] J. Deutsch, M. Borbely, J. Filler, K. Huhn, and P. Guarrera-Bowlby, "Use of a low-cost, commercially available gaming console (wii) for rehabilitation of an adolescent with cerebral palsy," Physical Therapy, vol. 88 , pp. 1196 - 1207, Oct. 2008.

[4] D. Tan, W. Chen, and M. Rauterberg, "Sleeping posture analysis of economy class aircraft seat," in the World Congress on Engineering, pp. $55-62$, Jun. 2009.

[5] M. M, H. AC, and S. JR., "Effect of work station design on sitting posture in young children," Ergonomics, vol. 4, pp. 1932 - 1940, Sept. 1995.

[6] K. Kamiya, M. Kudo, H. Nonaka, and J. Toyama, "Sitting posture analysis by pressure sensors," in International Conference on Pattern Recognition, pp. 155 - 162, Dec. 2009.

[7] http://www.tekscan.com/.

[8] J. Meyer, P. Lukowicz, and G. Troster, "Textile pressure sensor for muscle activity and motion dection," in International Conference on Wearable Computing, pp. 34 - 41, Sept. 2007.

[9] J. Farringdona, A. J. Moorea, N. Tilburyb, J. Churchb, and P. D. Biemond, "Wearable sensor badge and sensor jacket for context awareness," in International Symposium on Wearable Computer, pp. 107 113, Sept. 1999.

[10] W.-C. Du, X.-M. Tao, and H.-Y. Tam, "Effect of work station design on sitting posture in young children," IEEE Phontonics Technology Letter, vol. 1, pp. 105 - 107, Jan. 1999.

[11] J. Meyer, B. Arnrich, J. Schumm, and G. Troster, "Design and modeling of a textile pressure sensor for sitting posture analysis," IEEE Sensor Journal, vol. 10, pp. 1391 - 1398, Aug. 2010.

[12] C. Mattmann, O. Amft, H. Harms, G. Troster, and F. Clemens, "Recognizing upper body postures using textile strain sensors," in IEEE International Symposium on Wearable Computers, pp. 463-466, OCT 2007.

[13] E. Mitchell, S. Coyle, N. Connor, and D. Diamond, "Breathing feedback system with wearable textile sensors," in IEEE Conference on Body Sensor Network, pp. 56 - 61, Jun. 2010.

[14] L. Shu, T. Hua, Y. Wang, Q. Li, F. Dong, and X. Tao, "In-shoe plantar pressure measurement and analysis system based on fabric pressure sensing array," IEEE Transactions on Information Technology in Biomedicine, vol. 14, pp. 767 - 775, May. 2010.

[15] http://www. eeonyx.com/.

[16] M. Rofouei, W. Xu, and M. Sarrafzadeh, "Computing with uncertainty in a smart textile surface for object recognition," in IEEE International Conference on Multisensor Fusion and Integration for Intelligent Systems, pp. 50 - 65, Sept. 2010. 

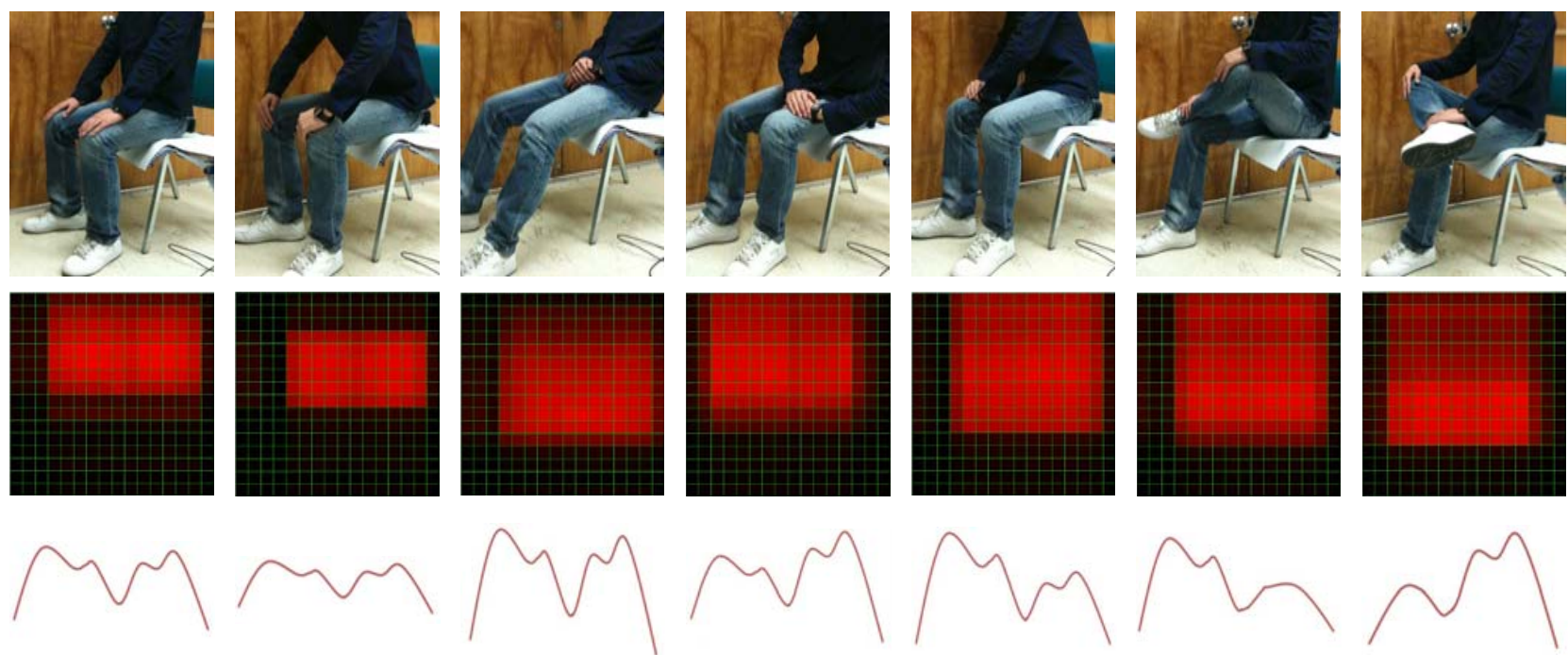

Fig. 8. Sitting Posture Processing: seven sitting postures(top) are evaluated and the each eTextile pressure map (middle) is transformed to corresponding pressure profile sequence (bottom)

[17] D. Li and S. Simske, "Shape retrieval based on distance ratio distribution," in HP Technology Report, pp. 56 - 78, Sept. 2002.

[18] M. Zhu, A. Martinez, and H. Tan, "Template-based recognition of static sitting postures," in Computer Vision and Pattern Recognition Workshop, pp. 50 - 53, Jun. 2003.

[19] N. Friedman and M. Goldszmidt, "Building classifiers using bayesian networks," in AAAI Conference, pp. 1277 - 1284, Sept. 1996.

[20] E. Keogh and C. Ratanamahatana, "Exact indexing of dynamic time warping," in ACM International Conference on Very Large Data Bases, pp. $92-111$, Sept. 2002.

[21] E. Keogh, L. Wei, X. Xi, S. hee Lee, and M. Vlachos, "Lb keogh supports exact indexing of shapes under rotation invariance with arbitrary representations and distance measures," in ACM International Conference on Very Large Data Bases, pp. 56 - 78, Sept. 2006. 